\title{
Applicability of the time-temperature superposition principle in modeling dynamic response of a polyurea
}

\author{
J. Zhao • W.G. Knauss • G. Ravichandran
}

Received: 21 September 2007 / Accepted: 28 November 2007 / Published online: 24 January 2008

(C) Springer Science+Business Media B.V. 2008

\begin{abstract}
This paper addresses the applicability of the Time-Temperature Superposition Principle in the dynamic response of a polyurea polymer at high strain rates and different temperatures. Careful and extensive measurements in the time domain of the relaxation behavior and subsequent deduction of a master-relaxation curve establish the mechanical behavior for quasistatic deformations over a time range of 16 decades. To examine its validity in a highly dynamic environment, experiments with the aid of a split Hopkinson (Kolsky) pressure bar are carried out. The use of a two-material pulse shaper allows for stress equilibrium across the specimen during the compression process, to concentrate on the initial, small deformation part that characterizes linearly viscoelastic behavior. This behavior of polyurea at high strain rates and different temperatures is then investigated by comparing results from a physically fully three-dimensional (axisymmetric) numerical model, employing the quasistatically obtained properties, with corresponding Hopkinson bar measurements. The experimentally determined wave history entering the specimen is used as input to the model. Experimental and simulation results are compared with each other to demonstrate that the Time-Temperature Superposition Principle can indeed provide the requisite data for high strain rate loading of viscoelastic solids, at least to the extent that linear viscoelasticity applies with respect to the polyurea material.
\end{abstract}

Keywords Viscoelasticity · Time-temperature superposition · Master curve $\cdot$ High strain rates $\cdot$ Hopkinson bar

\section{Introduction}

The behavior of polymers depends strongly on the time-rates of their deformation, on temperature, as well as on (high) pressure (Tschoegl et al. 2002). This time dependence is understood to stretch over as many as 20 decades, not all of which can be accessed realistically

J. Zhao · W.G. Knauss $(\bowtie) \cdot$ G. Ravichandran

Graduate Aeronautical Laboratories, California Institute of Technology, Pasadena, CA 91125, USA

e-mail:wgk@caltech.edu 
at any one temperature. However, half a century of work involving viscoelastic characterization of polymers has convinced the polymer physics community through measurements that a special relation exists between the time scale and the use temperature. In particular, it has been accepted for a long time that, apart from other small temperature effects related to rubber elasticity concepts (Treloar 1975) that, when plotted on a base-10 logarithmic time basis the physical characteristic response functions - the relaxation modulus or the creep compliance-translate along the log-time axis by an amount that is a unique function of the temperature. Generally, "colder temperatures" effect a shifting towards longer times, while the converse is true for "higher temperatures". This phenomenon, usually referred to as the time-temperature shift principle, is well accepted and has been reconfirmed many times for amorphous polymers at temperatures above the glass transition temperature. ${ }^{1}$

Because the time scales of laboratory tests are typically limited to a few decades ${ }^{2}$ test results obtained at different temperatures can be used together with this translating or shifting process to generate response curves that extend over many more decades than the laboratory tests would allow per se. The result of such a composition process is usually termed a master curve. An example of this process leading to a master curve that extends over many decades of time which were not physically encountered in the laboratory is delineated later in this paper. Because such measurements are universally made in a quasistatic time frame measured in seconds, minutes and hours, the applicability of the master curve to the much shorter time frames as encountered in wave propagation problems has been repeatedly called into question.

One may argue that wave propagation phenomena relate to inertial effects rather than material behavior as such, so that the same material response characteristics should apply with equal validity to stress and deformation states generated quasistatically or via inertial conditions. However, that argumentation has never quite satisfied investigators whose main efforts concentrate on dynamics problems. As a result, the applicability of data acquired through quasistatic tests and "extrapolated via the time-temperature superposition process" to engineering problems involving much shorter time scales has long been in question. It is the purpose of this publication to address that issue. As a corollary it would seem that if one can show that the time-temperature reduced data is applicable in dynamic situations for this particular material, there is a great likelihood - if not a guarantee- that the same should be true for other amorphous ${ }^{3}$ polymers that obey the time-temperature equivalence principle.

This paper is organized into nine sections, with the remaining ones covering the topics General comments (Sect. 2), Quasistatic relaxation behavior (Sect. 3), Dynamic experimental arrangements (Sect. 4), Precision of the experimental method for linearly viscoelastic response, including pulse-shaping (Sect. 5), Computer simulation model, (Sect. 6), Comparison of experimental and computed dynamic responses (Sect. 7), Summary (Sect. 8) and References.

\footnotetext{
${ }^{1}$ This temperature is a material characteristic that determines the transition between rubber-like behavior and the (more) rigid state of the polymer.

${ }^{2}$ Sometimes investigators measure polymer creep or relaxation over as short a time scale as 1.5 to 2 decades; this is not a recommended practice, since 3 to four decades provides considerably more definition for the mechanical characterization process for constructing the master curve by time-temperature shifting.

${ }^{3}$ This material is actually not a completely amorphous material, since there exists a small amount of crystalline domains. However, these relatively rigid phases do not come into play in the temperature domain under study and should, therefore, not compromise the results.
} 


\section{General comments}

We have limited the investigation to the small deformation range, so that linearly viscoelastic behavior can be inferred. While many engineering applications are not fully served by this deformation range, there is a cogent reason for this restriction. Inasmuch as we are interested in assessing the applicability of properties determined quasistatically by time-temperature superposition so as to be used in dynamic applications, we must adhere to the rules under which the time-temperature superposition is commonly practiced. Relaxation and creep properties are consistently determined over a large temperature-reduced time scale for the small deformation range (linear material response). Because the viscoelastic behavior of all polymers is poorly understood in the non-linear range, we cannot afford to mix this uncertainty into the examination of whether the temperature-reduced time scales are applicable in dynamic situations. We can only hope, that if an equivalence can be established for small deformations, then any extension of research to the nonlinear behavior range(s) may experience fewer roadblocks.

The interpretation of experimental results is always subject to the quality of data. With that recognition in mind considerable effort was expended on as careful a material characterization as was feasible with the existing equipment. To the extent that equipment improvements could be managed economically, such improvements were incorporated. A serious challenge arose because of the relatively small size of the (compression) specimens, in addition to the desire to remain within the linear response realm, so that small deformations needed to be resolved with considerable precision. In the near-rubbery domain, where low stiffness dominates, strains on the order of $3.8 \%$ or less were deemed sufficient, while at the higher rates when the material responds more stiffly strains of about $2 \%$ or less were considered adequate. One reason why the higher strains in the rubbery domain were allowed was that the increased strains also increased the stress level which improved the signal-to-noise ratio of the load cell signal: loads measured at elevated temperatures tended to be small so as to fall into the lower measuring range of the load cell. ${ }^{4}$ Finally, existing thermal conditioning facilities were limited to cooling specimens to the glass transition temperature of polyurea. For the present study, this temperature range was considered sufficient, because the time-temperature superposition principle rests on its physically and theoretically most coherent foundation/explanation there.

In determining the relaxation modulus of a viscoelastic material under quasistatic conditions one makes use of simple relations describing homogeneous stress and strain fields in equilibrium situations such that the modulus property can be extracted readily. For example, the determination of the relaxation modulus needs, in principle, "only" the prescription of a fixed tensile or compressive strain and the measurement of the force (and specimen cross sectional area) required to maintain that deformation. Such a simple possibility does not exist with Hopkinson bar measurements, even if the specimen is loaded so that it may be considered to be temporarily in a state of equilibrium. To illustrate this observation the reader may, at this point, refer to Fig. 14 below which demonstrates the strain rate history in a Hopkinson bar measurement. For the purpose of viscoelastic material characterization it is thus nearly impossible to prescribe a simple load history in Hopkinson bar equipment (e.g. a constant strain rate history) since the striker bar contact and pulse shaper impose their own constraints. In principle one could — and we do-measure the incident force history and the resulting strain history for deducing the transfer function via an integral equation, the result

\footnotetext{
${ }^{4}$ A newly purchased, low capacity load cell ultimately proved to lack sufficient precision.
} 
of which would be the relaxation modulus or the creep compliance. However, for an arbitrary, if measured, load or deformation history this property extraction could be tantamount to a trial and error fitting of a range of viscoelastic functions so as to best match the results of such correlated measurements.

Because the trial and error fitting could be a daunting task one might argue that much effort can be saved if one starts out with the quasistatically determined master curve as a "first guess" and then provide variations around this function to make the Hopkinson bar measurements correspond with each other. On the other hand, if, under that scenario, the master curve were to satisfy the dynamically measured correlation between force and deformation without further correction, this observation would be equivalent to having shown that the quasistatically determined master curve does, indeed, represent the material behavior under dynamic conditions. This is the approach we follow in the subsequent work: After documenting derivation of the relaxation master curve through low-speed test procedures, we make use of this material function to compute the response of the Hopkinson bar specimen under dynamic conditions, and then compare these computations with the measured laboratory counterpart.

\section{Quasistatic relaxation behavior}

Because this study was part of a larger effort devoted to compressive stress fields of elastomers we chose to determine the relaxation behavior under (uniaxial) compression, though for linearly viscoelastic behavior no distinction arises with regard to the tensile or compressive nature of the imposed deformation. Specimens were used having cross sections of $14 \times 14 \mathrm{~mm}^{2}$ and $30 \mathrm{~mm}$ in length.

Preliminary tests on a servo-hydraulic materials testing system (MTS) invariably showed data irregularities in the start-up phase, though a ramp history with a $10 \mathrm{sec}$ ramp time instead of one of $1 \mathrm{sec}$ duration improved the recovery of the relaxation modulus. Two paradigms were employed to reduce errors and noise effects. Because some of the force transients were traced to the gradual compression and seating between specimen and testing machine platens, both the specimen and the platens were lapped and polished-the specimen in a special holding fixture - to achieve surfaces that were parallel to within $1 \mathrm{mi}$ cron per mm across the specimen diameter. Moreover, to minimize the last vestiges of such undesirable errors the specimens were pre-compressed through a displacement of 0.02 to $0.04 \mathrm{~mm}$, with a subsequent rest period of at least one hour to allow sufficient time for the associated relaxation to a ("rubbery") steady stress before imposing the ramp history (as the dominant, additive load history). To assure acceptable data consistency commensurate with the small displacements corresponding to the small strains, the oil in the test frame was allowed to achieve a constant temperature of at least $40^{\circ} \mathrm{C}$ during two hours of warm-up of the MTS system. The temperature control unit was allowed to reach its pre-set temperature during at least a one-hour start-up period followed by a one hour thermal equilibration of the specimen. To eliminate any potential thermally induced preload, the relative displacement between the load-free ends of the test frame were monitored with an LVDT, so that preloading would occur only after that relative displacement was found to be non-detectable. During testing, the temperature varied within a range of no more than $\pm 0.5^{\circ} \mathrm{C} .^{5}$

\footnotetext{
${ }^{5}$ For additional detail the reader may wish to consult the reference by Knauss and Zhao (2008) which deals with enlarging the time scale over which data can be recovered in a ramp test.
} 
Fig. 1 Relaxation behaviors at different temperatures

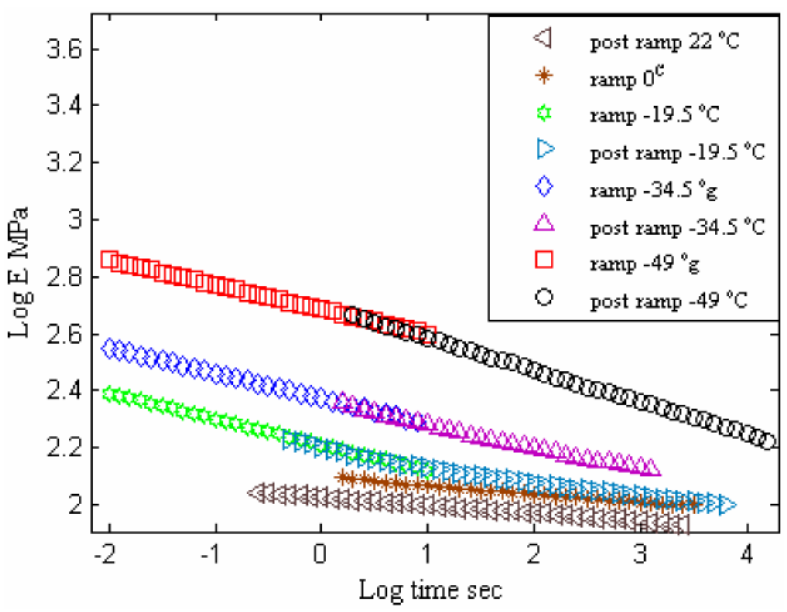

Fig. 2 Master curves at $0^{\circ} \mathrm{C}$ for two specimens generated from the same material batch $\left(a_{\mathrm{T}}=1\right.$ at $0^{\circ} \mathrm{C}$ )

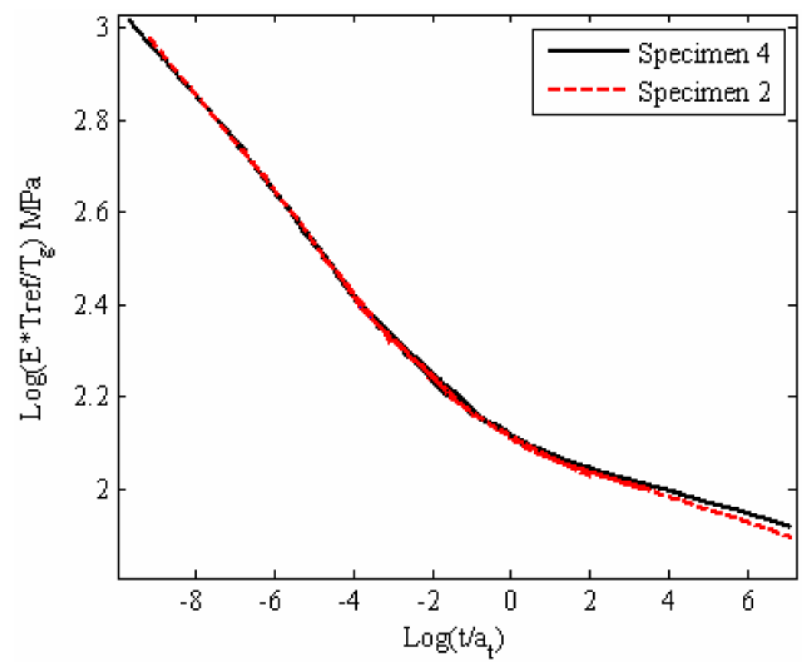

Because of the small specimen dimensions and the relatively long specimen support structure of the test frame which extended into the thermal control chamber, the thermally induced dimensional changes of this rod connection could result in significant errors in the strain prescription. For this reason that support was manufactured from Invar (an iron-nickel possessing very small thermal expansivity), which design feature virtually eliminated that possible error source.

Figure 1 records the relaxation data as measured at various temperatures in a ramp strain history ${ }^{6}$ these data were then reduced via the standard time temperature shift process, including the correction in the modulus for the rubber-elastic response to temperature (modulus $\sim$ absolute temperature) to produce Fig. 2. Figure 2 incorporates the results of tests on two geometrically identical specimens, each one of which was, however, cut from a different

\footnotetext{
${ }^{6}$ Data from both the constant strain rate and constant strain portions were used. The figures refer to the
} constant strain rate portion as the "ramp" part and to the constant strain portion as the "post-ramp" history. 
Fig. 3 Shift factor as a function of temperature

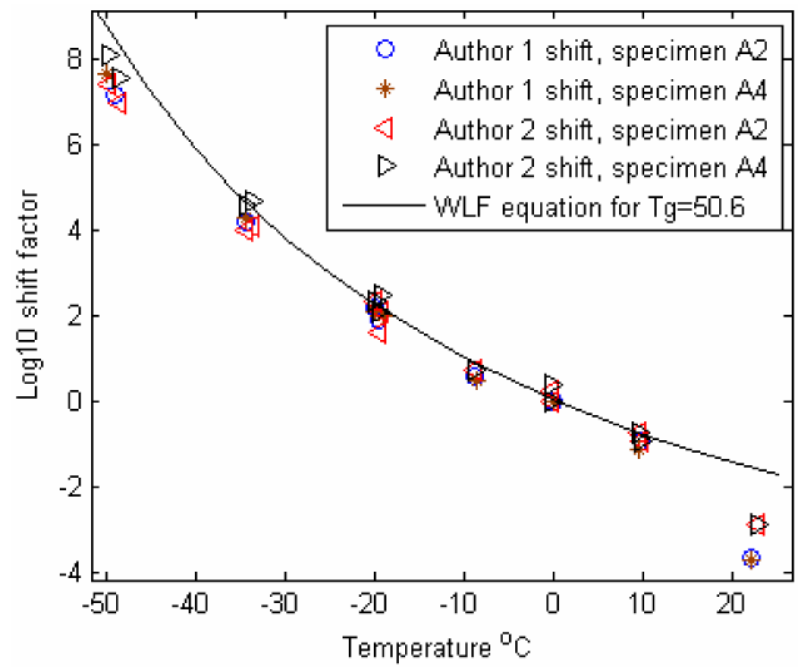

location in the raw material sheet supplied. The first two authors each performed the shifting process separately and independently, with identical shift results as shown in Fig. 3. The WLF equation (Williams et al. 1955) referenced in the figure was fitted in the most often used form of

$$
\log a_{T}=\frac{-8.86\left(T-T_{S}\right)}{101.6+\left(T-T_{S}\right)}
$$

where the reference temperature $T_{s}$, often valued at $50^{\circ} \mathrm{C}$ above the glass transition temperature, was taken as unity. This places the glass transition temperature for the polyurea material at around $-49^{\circ} \mathrm{C}$.

\section{Dynamic experimental arrangements}

To access the high strain rate domain we made use of a split Hopkinson (Kolsky) pressure bar shown schematically in Fig. 4. The time scale of these measurements is on the order of $10^{4}$ to $10^{8}$ times shorter than those employed in the quasistatic tests. Split Hopkinson bars are typically used for non-dispersive materials possessing considerably higher stiffness than the polyurea material at hand. As a consequence the deformation and stress levels tend to be rather large and not in keeping within the small deformation goals intended for study here. For this reason it will be necessary to carefully assess the precision to be expected from this device at the load and strain levels of interest.

The split Hopkinson pressure bar consists of a striker bar, an incident bar and a transmission bar of $250 \mathrm{~mm}, 2200 \mathrm{~mm}$, and $1500 \mathrm{~mm}$ lengths, respectively; all are of 7075-T6 Aluminum and possess a common diameter of $12.7 \mathrm{~mm}$. Two X-cut quartz gages of $12.7 \mathrm{~mm}$ diameter and $0.254 \mathrm{~mm}$ thick were mounted coaxially and close $(10 \mathrm{~mm})$ to the specimen contact surfaces on the incident and transmission bars for recording the longitudinal force (stress) on both cross sectional specimen surfaces. In addition, $1000 \Omega$ strain gages with a gage factor of 3.27 were bonded to the incident and transmission bars in the locations shown in Fig. 4 to track the strain signals. Because the specimen impedance was typically small the transmitted signal tended to be weak. Therefore, two strain gages were attached on diametrically opposite sides of the transmission bar, which were then connected as additive 


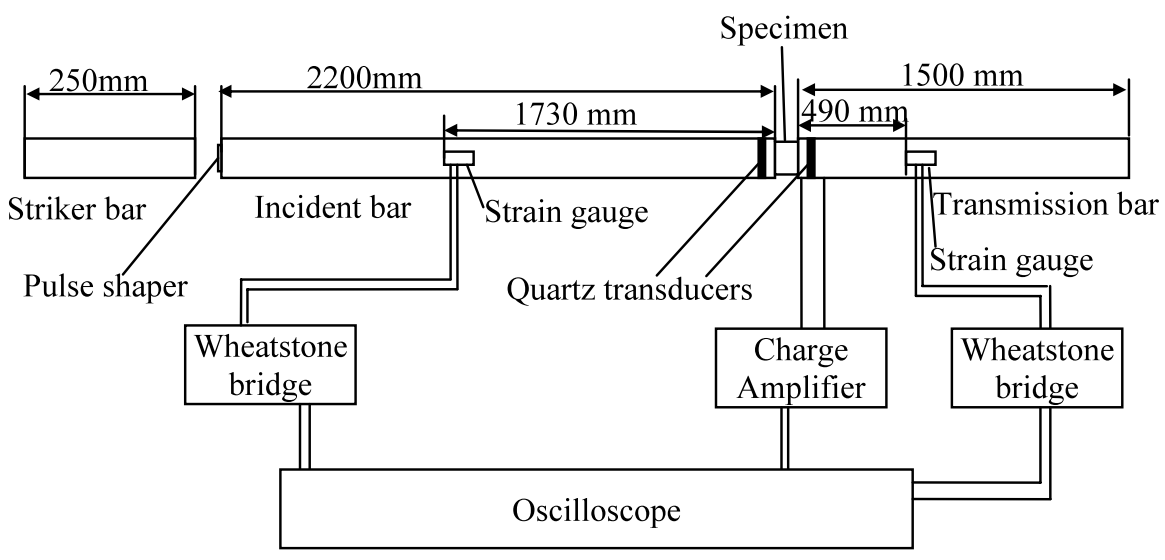

Fig. 4 Split Hopkinson bar arrangement

branches in the Wheatstone bridge circuit to double the strain signal and thus to improve the signal-to-noise ratio.

\subsection{Specimen preparation and bar alignment}

Cylindrical specimens were used which were typically $3 \mathrm{~mm}$ thick and $9 \mathrm{~mm}$ in diameter, though some specimens were cylinders of $6 \mathrm{~mm}$ diameter. Special precautions were taken in preparing these for the Hopkinson bar measurements. A major inaccuracy can derive from a less than perfect contact at the interfaces between the specimen and the incident and transmission bars. Of foremost importance is the elimination or at least reduction of an initially partial contact across the end surfaces of the specimen. In traditional Hopkinson bar measurements this issue is of relatively minor importance since the deformations encountered are usually so large that perturbations of this kind of magnitude play no significant role. However, to address the material linearity of the deformation process this geometric (contact) issue required considerable development effort resulting in the design of a special lapping fixture that allowed generation both of flat surfaces and their parallel alignment to within $0.01 \mathrm{~mm}$ across the specimen diameter $(0.00011 \mathrm{rad}=0.064$ degrees $)$. To convey the sensitivity of this alignment of the bar ends relative to the specimen surfaces we note that this would correspond to a misalignment similar to bending the roughly 2-meter long transmission bar to a central deflection of less than half a millimeter. Thus, to assure near-perfect alignment of the incident and transmission bars, new and straight bars were specifically manufactured. A satisfactory alignment was then always guaranteed if, in the absence of a specimen, the wave transmission across the bar faces produced no more than a "blip" in the signals, the amplitude of which was small compared to the measurement signals in our experiments. Such alignment checks were carried out routinely during the sequence of the measurements reported here. An example of the signals generated with the aid of the composite pulse shaper, discussed later, and in the absence of a specimen is shown in Fig. 5. In this case all traces have been shifted to the same time frame to ease the comparison of the various signals.

In view of the subsequent modeling analysis thought must be given to the boundary conditions at the interfaces between the specimen and the incident/transmission bars. To 
Fig. 5 Signals (in the absence of a specimen) from all gages in the Hopkinson bar generated with a composite pulse shaper consisting of polyurea $(1.32 \mathrm{~mm}$ thick, and $8.11 \times 8.17$ laterally) and 16 layers of paper

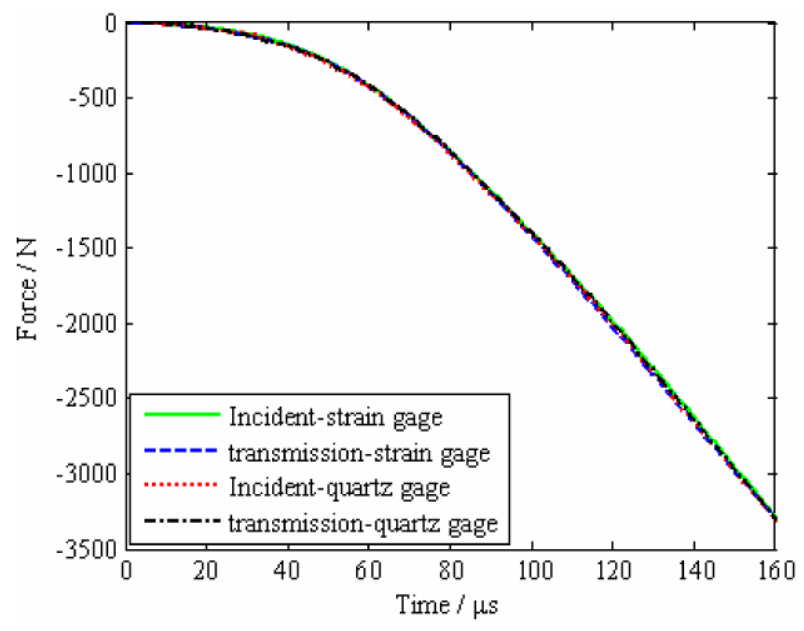

eliminate/reduce the lateral displacement constraint derived from friction at these interfaces a lubricant was used that operated also satisfactorily at the low test temperatures. ${ }^{7}$

\subsection{Temperature control and effect on transducers}

To investigate the dynamic response at low temperatures, a small temperature control chamber was constructed from half inch thick PMMA plates to fit into the Hopkinson bar environment as shown in Fig. 6. The specimen was cooled by allowing the evaporated nitrogen to flow from the tank directly into the chamber via a control valve. To avoid the purchase or construction of a thermal control system for the few tests envisioned, it was more resource efficient to implement manual control. This approach was quite feasible since temperature control was not required to produce a particularly precise test temperature, since the test duration was very short. Use of direct nitrogen insertion into the chamber obviated the problem of introducing (invariably) moist air into the sub-freezing environment with the attendant frosting of the chamber that obscured visual tracking of the set-up inside. To assist in maintaining a sufficiently stable temperature evolution, four aluminum blocks were located in the chamber close to the specimen to act as a thermal mass.

The flow valve was controlled manually for a cooling rate of about $-1^{\circ} \mathrm{C} /$ minute. The human control loop was guided by four thermocouples, of which one was located next to the incident bar and close to a quartz gage, so as to track the interface temperature, one was in close proximity to the specimen, and two were attached to a dummy specimen, $2 \mathrm{~mm}$ thick with metal ends, so as to simulate the real specimen, except that the environmental thermocouples were substituted by one that penetrated into the polymer of the dummy specimen, and the other into one metal end. The latter dummy assembly was also located close to the test specimen. While the heat flow into and out of the four thermocouples was usually not coordinated in phase, their temperature differences varied typically by no more than 1 to $3^{\circ} \mathrm{C}$. We relied most heavily on the thermocouple embedded in the dummy specimen for ascertaining the test temperature.

In view of the results discussed in Sect. 7 it is important to recognize the following facts: As temperatures approaching the major glass transition, the shift factor changes rather

\footnotetext{
${ }^{7}$ Low temperature ZP Grease, operational to $-200^{\circ} \mathrm{C}$, and manufactured by MK Impex, Canada.
} 
Fig. 6 Temperature control chamber

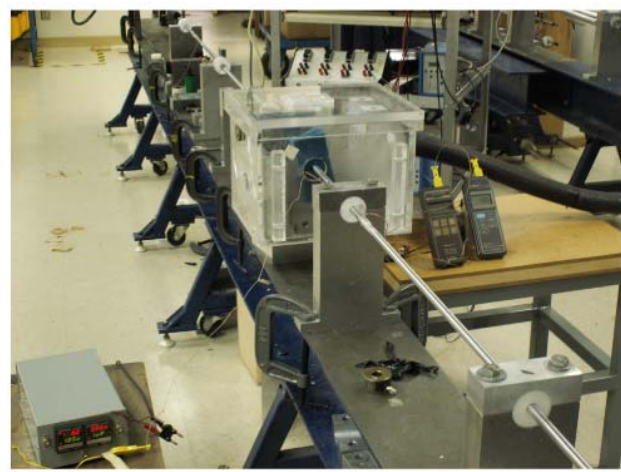

(a) Chamber and Hopkinson bars

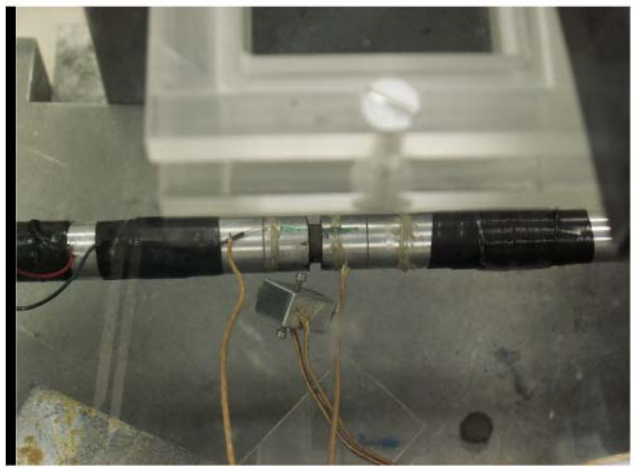

(b) Specimen inside the control chamber

strongly with temperature and thus an uncertainty of 1 to $3^{\circ} \mathrm{C}$ induces a more significant uncertainty in the time dependence of the relaxation modulus. A consequence of this disproportionately large uncertainty is that an "experimentally indicated" temperature may lead to a computed dynamic response that is not "identical" with the measured one. On the other hand, if the measured response falls within the range of the computed responses based on the extremes of the thermal uncertainty band, one may at least conclude that the quasistatically determined properties do not invalidate their application to dynamic situations.

The question arises as to how the thermal variations affected any transducer outputs. Because the strain gages were not contained in the temperature control chamber they remained at room temperature and that issue did not arise, even though these gages were temperature corrected. The manufacturer of the quartz gages could not provide any reliable data on their thermal performance so that calibration tests were called for. It turned out, however, that in the temperature range of interest in this work, no sensitivity to temperature changes could be detected.

\section{Precision of the experimental method for linearly viscoelastic response including pulse-shaping}

It is well known that experiments with Hopkinson bars are normally intended to access high strain rates and large deformations. However, because of our interest in linearly viscoelastic 
response and correspondingly small response signals, the performance of this experimental tool needs to be carefully assessed and controlled.

Before showing the results of this process we comment first on the way in which wave propagation data is presented here: Because the strain and quartz gages are located at different stations along the length of the Hopkinson bar the time histories of their signals, specifically of the wave arrival times, will be time-spaced according to their locations. However, to facilitate signal comparison we display the signals for the incident bar time-shifted so as to refer each one to its respective arrival time; they appear, therefore, as if they were to originate at the same time. The same applies to the signals from the transmission bar, though the time differential between the incident and the transmission bars is maintained. This operation can be performed with some precision because the distances between the gages and the attendant wave propagation speeds are well known.

The equations typically used for Hopkinson bar studies require that the time history be such that the specimen is essentially in a homogeneous state of stress and deformation. In contrast to when wave motion dominates within the specimen, we refer to this state also as "a dynamic equilibrium state" or simply "equilibrium state". Even if that condition is conceptually not an absolute requirement for our study - in view of the fact that comparison with computational modeling is involved-its use simplifies the analysis and interpretation of the dynamic signals markedly, if for no other reason than to "trouble-shoot" the measurements. With the relatively high importance of precision measurements required in this study, we found this latter aspect very important. To achieve specimen equilibrium one typically controls the shape of the incident wave by "pulse shaping" through the insertion of a suitably configured material element between the striker and incident bars. We are well aware that this pulse shaping comes at the expense of somewhat reduced strain rates, accounting for almost a decade in the short-time frame, yet considered this sacrifice worth while to guarantee the requisite precision of the measurements.

Under the equilibrium conditions the pertinent equations are, with the subscript definitions $i=$ incident, $r=$ reflected $t=$ transmitted, and $s$ referring to the specimen,

$$
\begin{aligned}
\varepsilon_{s} & =\frac{2 c_{0}}{l_{s}} \int_{0}^{t}\left(\varepsilon_{i}-\varepsilon_{t}\right) d \tau=-\frac{2 c_{0}}{l_{s}} \int_{0}^{t}\left(\varepsilon_{r}\right) d \tau \\
\dot{\varepsilon}_{s} & =\frac{2 C_{0}}{l_{s}}\left(\varepsilon_{i}-\varepsilon_{t}\right)=-\frac{2 C_{0}}{l_{s}} \varepsilon_{r} \\
\sigma_{s} & =\frac{E A}{A_{s}}\left(\varepsilon_{i}+\varepsilon_{r}\right)=\frac{E A}{A_{s}} \varepsilon_{t}
\end{aligned}
$$

where we remind the reader that elastic relations apply since the quantities refer all to measurements deduced from the strain and quartz gages attached to the elastic aluminum bars.

The desire or need to deal with a specimen in equilibrium generates a significant challenge that derives from the large impedance mismatch between the specimen and the aluminum bars. To deal with a similar situation involving materials of relatively low rigidity, Chen et al. (2002) introduced the pulse shaping technique, which seems to work quite well for polymeric materials under large deformations. For the present purposes we have explored pulse shapers of different materials, for example copper, various polymers and wood, but obtained unsatisfactory results for reaching stress equilibrium when loading forces were smaller than about a hundred Newtons. Chen et al. (2002) encountered the same problems but were able to ignore these issues because they were minor perturbations on the larger deformation results that were of interest to them. Through a trial-and-error process we found that employing a composite pulse shaper consisting of a combination of polyurea (1 to $2 \mathrm{~mm}$ 
Fig. 7 Room temperature record of forces at the quartz gages for a pulse shaper of polyurea only

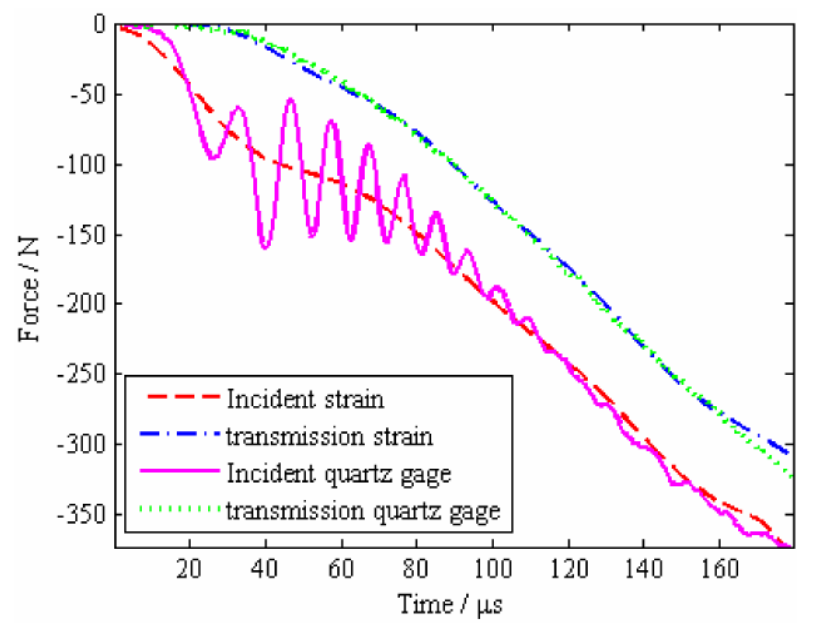

thick) and 16 sheets of paper towe ${ }^{8}$ in tandem provided a highly acceptable shape with a minimal sacrifice of short time/high rate performance.

Figure 7 shows the force histories deduced from the wire-strain and quartz gages, when only a polymer pulse shaper was employed. Here the notation "incident strain" in the caption of that figure signifies that the force was deduced from the incident and reflective waves (4), both of which are recorded by the incident strain gage. The "transmission strain" in Fig. 7 denotes that the force is deduced from the transmission wave recorded by the transmission strain gage. Also, Q1 and Q2 refer to the force histories recorded by the incident and the transmission quartz gages, respectively. We see that the force histories recorded by the incident strain gage and the incident quartz gage are consistent with each other except for the oscillation in the quartz gage signal, but they are not consistent with the force histories recorded by the transmission gages. However, when the composite pulse shaper is employed as illustrated in Fig. 8, one arrives at a very consistent agreement.

The result of this pulse shaping is illustrated in Figs. 9 and 10: both figures demonstrate the repeatability of separate test runs, with Fig. 9 indicating that a small temperature difference near $-22^{\circ} \mathrm{C}$ does not produce a measurable difference in the gage readings. By contrast, Fig. 10 demonstrates that significant temperature differences result in clearly distinguishable gage records. For completeness of presentation we show in these two figures extended time records which involve, however, also relatively large deformations of no direct interest in this study which, as rationalized in Sect. 2, is geared to small deformations. The reader should bear in mind that linearly viscoelastic behavior for this material is reasonably assured if stress levels are limited to 5 to $6 \mathrm{MPa}$ or to a force level on the order of $400 \mathrm{~N}^{9}$ which is the range exhibited in Fig. 8. Theses results demonstrate that much of the total test history typically involves relatively large stresses and strains exceeding the linear domain and that a comparison with linearly viscoelastic computations is expected to deviate progressively as nonlinear material behavior enters.

To apprise the reader of the special care in data evaluation required in the present case we comment on the special way in which data evaluation had to occur, which result from

\footnotetext{
${ }^{8}$ Georgia Pacific, Roll towel, part \# 26100.

${ }^{9}$ Maximal strains on the order of $3.5 \%$ at room temperature, smaller at lower temperatures, typically around $2 \%$.
} 
Fig. 8 Room temperature record of forces at the quartz gages for a pulse shaper of polyurea plus paper (pulse-shaper: polyurea $1.98 \times 8 \times 8 \mathrm{~mm}+16$ layers of tissue paper cylindrical specimen $\phi 9.5 \mathrm{~mm} \times 3 \mathrm{~mm})$
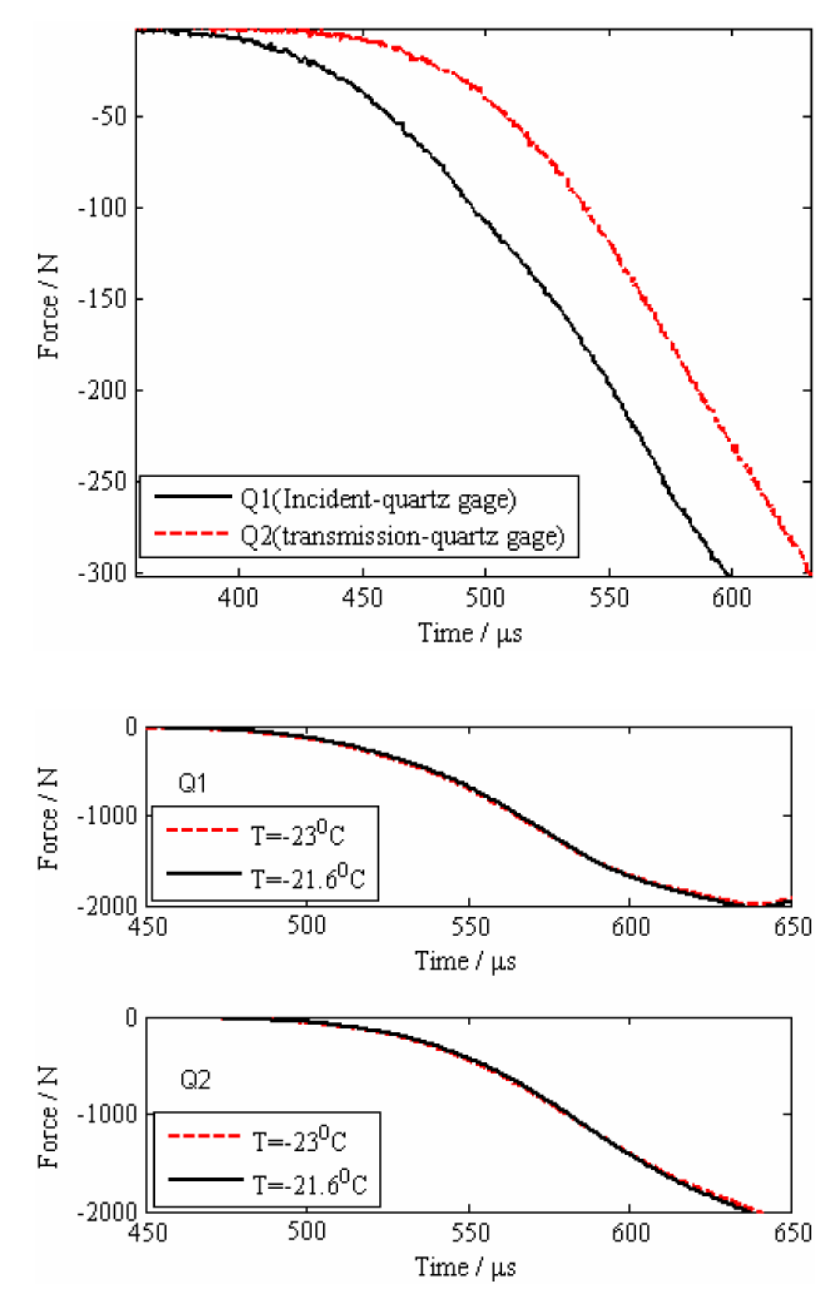

Fig. 9 Extended time history of quartz gage records on the incident bar (upper) and transmission bar (lower) for two specimens

the small deformation limitation in combination with the particular pulse shaping technique and the available equipment. These factors introduce a limitation not usually encountered in experiments involving larger deformations and better impedance matching. In the latter cases (2)-(3) usually allow the determination of the specimen strain from either the reflected signal or from the difference between the incident and transmitted signal. This alternative approach is not valid here. Because the striker bar does not rapidly rebound from the incident bar as a result of the special pulse shaper, the signal in the incident bar is not as "clean" as if such a separation had occurred on impact: it contains reflections within the striker bar, that are troublesome to isolate definitively for analysis purposes; consequently the reflected signal has not been used in this analysis.

Figure 11 demonstrates this by displaying the signal from the strain gage on the incident bar and that in the transmission bar. The part of the signal marked as "weak disturbance" is the low amplitude oscillation in the strain gage record due to the superposition of the less-than-perfect signal from the striker bar with the reflection at the incident-bar/specimen interface. The amplitude of this "disturbance signal" is small compared to the peak of the transmitted signal. However, when the strain in the specimen is derived from the signal 
Fig. 10 Extended time history of quartz gage records on the incident bar (upper) and transmission bar (lower) for the same specimen at three temperatures
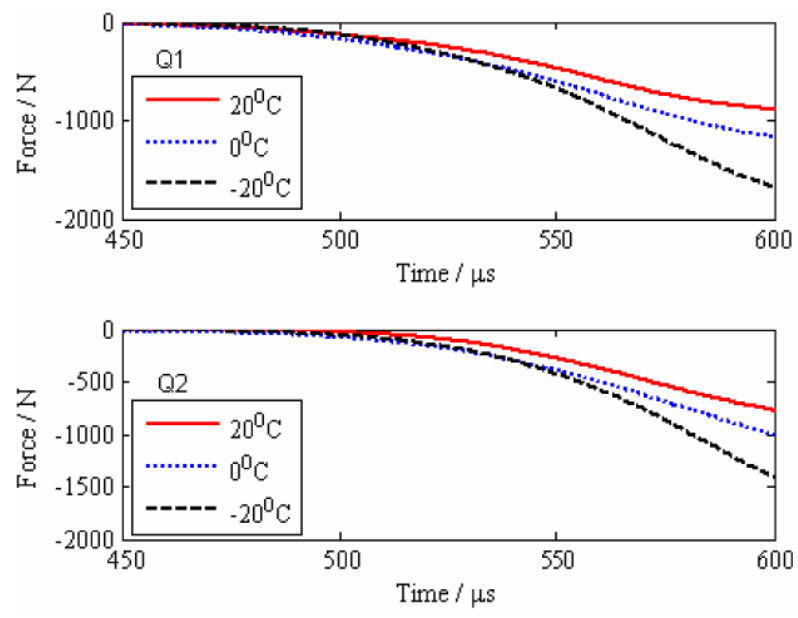

Fig. 11 Identification of a weak disturbance arising from the dispersion of the signal at the incident bar-specimen interface

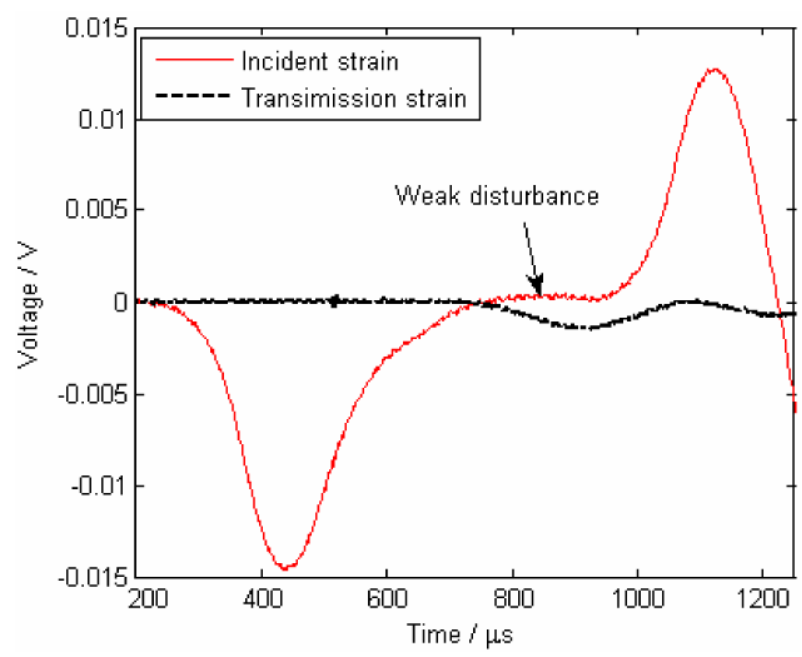

reflected at the incident specimen surface (see (2)) the "disturbance" amounts to a major component of the reflection signal. On the other hand, if one deals only with the-relatively small - transmission signal, that signal is much less encumbered by the "disturbance" and, as a consequence, yields a well defined measure of the specimen strain.

To illustrate this observation we anticipate results addressed in the next section and compare the computations of stress and strain histories in the specimen for the two ways of determining the specimen strain. Figure 12a shows the strain history in the specimen as determined from the reflected signal and from the difference between the incident and transmitted ones. The latter provides a much closer fit to the computed history, which is in close agreement with that derived from the incident/transmission combination. An identical result is shown for the stress history in Fig. 12b. Based on these results we adhere in the sequel to determining the specimen deformation and stress histories via the incident and transmitted signals. 


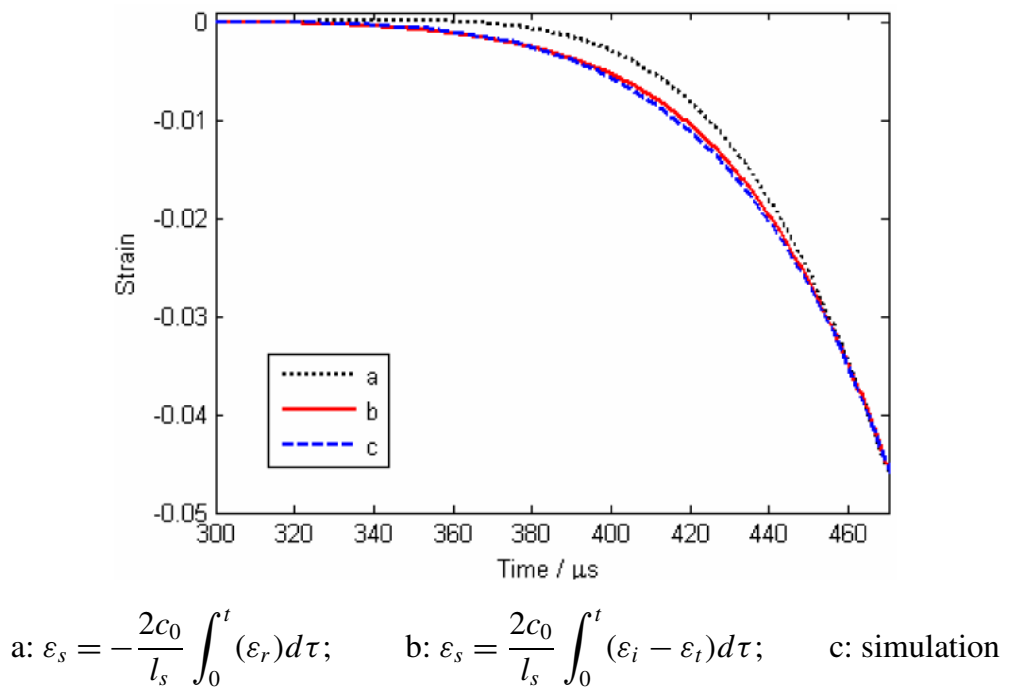

(a) Strain

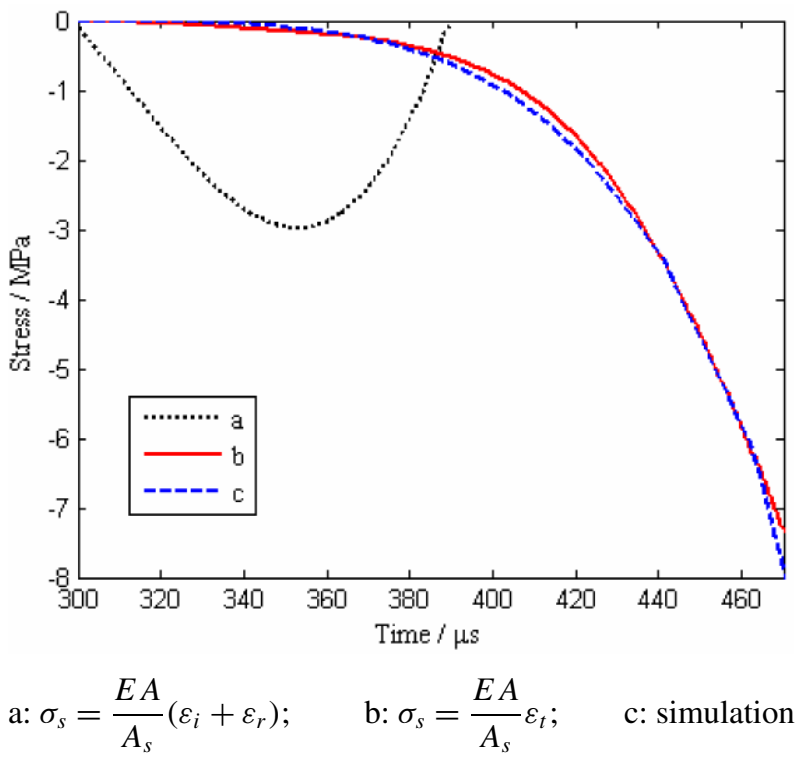

(b) Stress

Fig. 12 Improvement of data reduction through elimination of the reflected strain in favor of the incident and transmitted strains. Specimen and pulse shaping are the same as those in Fig. 11

\section{Computer simulation model}

As indicated in Sect. 2 the assessment of how well the quasistatically derived master curve represents the material response under dynamic conditions is best accomplished with the aid of a computational or simulation model. We have used the LS-Dyna 970 code which permits linearly viscoelastic material characterization in the form of relaxation or creep functions in 
shear ${ }^{10}$ on the one hand, and either a constant bulk modulus or a constant Poisson ratio, on the other. Because the bulk modulus typically varies by a much smaller amount over the viscoelastic transition range than the shear or uniaxial modulus it is a very good engineering approximation to represent the volumetric response by a time-independent bulk modulus. We note in passing that this approximation is more realistic in the transition range and still shorter times than choosing a constant Poisson characterization, because the latter choice links the bulk and shear (or uniaxial) modulus by a direct proportionality, a situation that is not generally supportable as the glassy range is approached. We choose as the best approximation the time-dependent, uniaxial (Young's) modulus and the corresponding shift factor as summarized above, and a constant bulk modulus of $263 \mathrm{GPa}^{11}$ (Chakkarapani et al. 2006). It turns out in the present case, however, that this choice of the bulk modulus also happens to allow the use of a constant Poisson's ratio in the time and temperature range of interest.

To guarantee that the code was able to reliably represent complex time histories of nontrivial initial/boundary value problems we compared its output to analytically tractable and closed-form solutions for viscoelastic problems using relatively simple material models (one or two relaxation times). Without recounting here all the details of these analyses, we simply report in the interest of brevity that in all test situations the code performed very well and in accordance with the anticipated results.

For the modeling process the Hopkinson bar test configuration was reproduced dimensionally except for the length of the incident and transmission bars: Because of the axisymmetric geometry the problems was formulated mathematically as two-dimensional, including the discontinuities at the transitions from the specimen to the adjacent bars. To allow for possible signals from these geometric changes the specimen discretization employed 15 uniformly spaced (ring) elements in the radial and 10 elements in the axial direction. The incident and transmission bars were modeled as 1500 and $1000 \mathrm{~mm}$ in length, respectively, each one divided into 12 coaxial rings of equal thickness and $0.53 \mathrm{~mm}$ in length, distributed uniformly along their lengths. The specimen and bar discretization was implemented via (axisymmetric) four-noded elements for a total element count of 60150, 150 of which are allocated to the specimen.

As input into the specimen we used the experimentally determined output of the strain gage on the incident bar. The properties for the aluminum bars used were: Young's modulus $(7075-\mathrm{T} 6$ aluminum $)=72 \mathrm{GPa}$, Poisson's ratio $=0.33$ and the density of $2.785 \mathrm{~g} / \mathrm{cm}^{3}$. The constitutive equation of the polyurea polymer was chosen in the form of a Prony series for the shear relaxation modulus as

$$
G(\xi)=G_{\infty}+\sum_{i=1}^{n_{G}} G_{i} e^{-\xi / \lambda_{i}^{G}} .
$$

\footnotetext{
${ }^{10}$ The code does not allow for the use of the uniaxial modulus. This was the function available for the polyurea, however. For this reason the shear modulus was derived from the unaxial modulus by the approximation valid in the rubbery domain, that $\mu(t)=E(t) / 3$. In the transition domain and at shorter times this approximation becomes less accurate; however, for the measured bulk modulus the deviation from reality is within the range of the experimental error.

${ }^{11}$ It is of parenthetical interest to note that the value of the bulk modulus is not very important, as long as it is large. Computations with values of $263 \mathrm{GPa}$ on the one hand, and $2 \mathrm{GPa}$ on the other rendered results that were indistinguishable within plotting accuracy.
} 
Fig. 13 Simulation model (only specimen-near portions of incident and transmission bars are shown; one half of axisymmetric configuration)

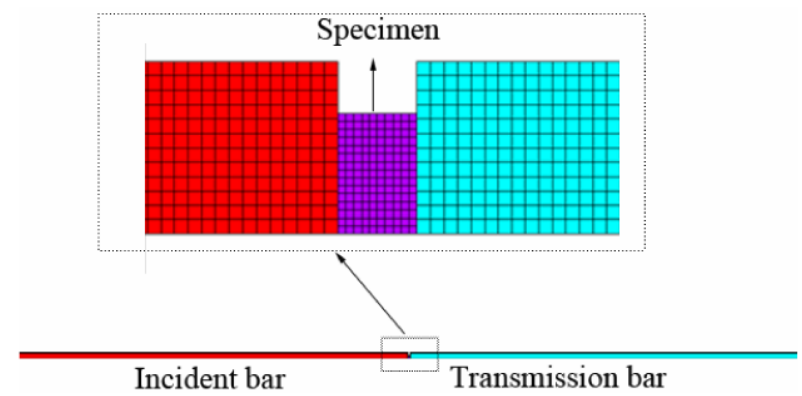

Fig. 14 Experimentally determined strain rate for conventional polyurea specimen $(6 \mathrm{~mm}$ dia $\times 2.65 \mathrm{~mm})$ under Hopkinson bar loading, as determined from

$\dot{\varepsilon}_{s}=\left(2 C / l_{s}\right)\left(\varepsilon_{i}-\varepsilon_{t}\right)$ where $l_{s}=$ specimen length $\varepsilon_{i}$ and $\varepsilon_{t}$ are the incident and transmitted strain signals, respectively

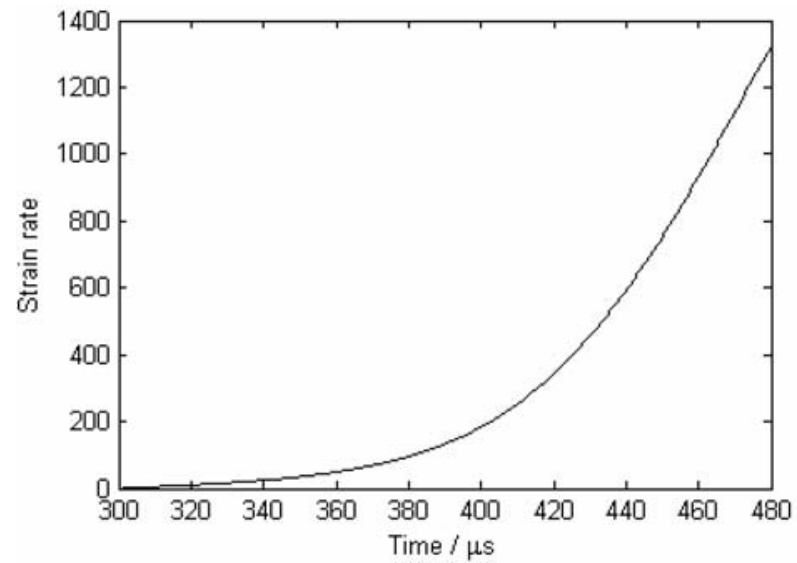

For current purposes it suffices to let $n_{G}$ be on the order of six with $\lambda_{i}^{G}$ suitably chosen to represent the time scale of the anticipated deformation and stress relaxation. For this limited time scale a representation with all relaxation times would yield no different results.

\section{Comparison of measured and computed dynamic responses}

Using the combined pulse shaper technique described above, polyurea specimens $3 \mathrm{~mm}$ thick and $9 \mathrm{~mm}$ in diameter were generally employed to explore their viscoelastic response. Figure 14 shows the strain rate in the compression process under Hopkinson bar loading, which varies continuously from near zero to around $10^{3} / \mathrm{s}$ before the strain reaches $4 \% \mathrm{com}$ pression. Higher strain rates are achieved at higher strains, but these take us out of the range of small-strain viscoelastic behavior. It was thus not possible to deduce the relaxation behavior from the Hopkinson bar data through a closed form solution. To follow a completely numerical-mathematical route which is possible, is, however, equivalent to the procedure we follow subsequently because we know the relaxation modulus from quasistatic measurements with the aid of the time-temperature super-position principle. If we assume for computational purposes that these data apply to the dynamic situation we can test that assumption by comparing the computations with the experimental results.

Figure 15 shows a comparison of the force recorded via the quartz gages and the simulation at the location of the quartz gages in the bars. The excellent agreement in the measured and computed force histories indicates that we can compute the counterparts to the dynamic 
Fig. 15 Comparison between dynamic experimental (quartz gage) and simulation results

Fig. 16 Comparison between the experimental and simulated stress-strain relation under dynamic loading
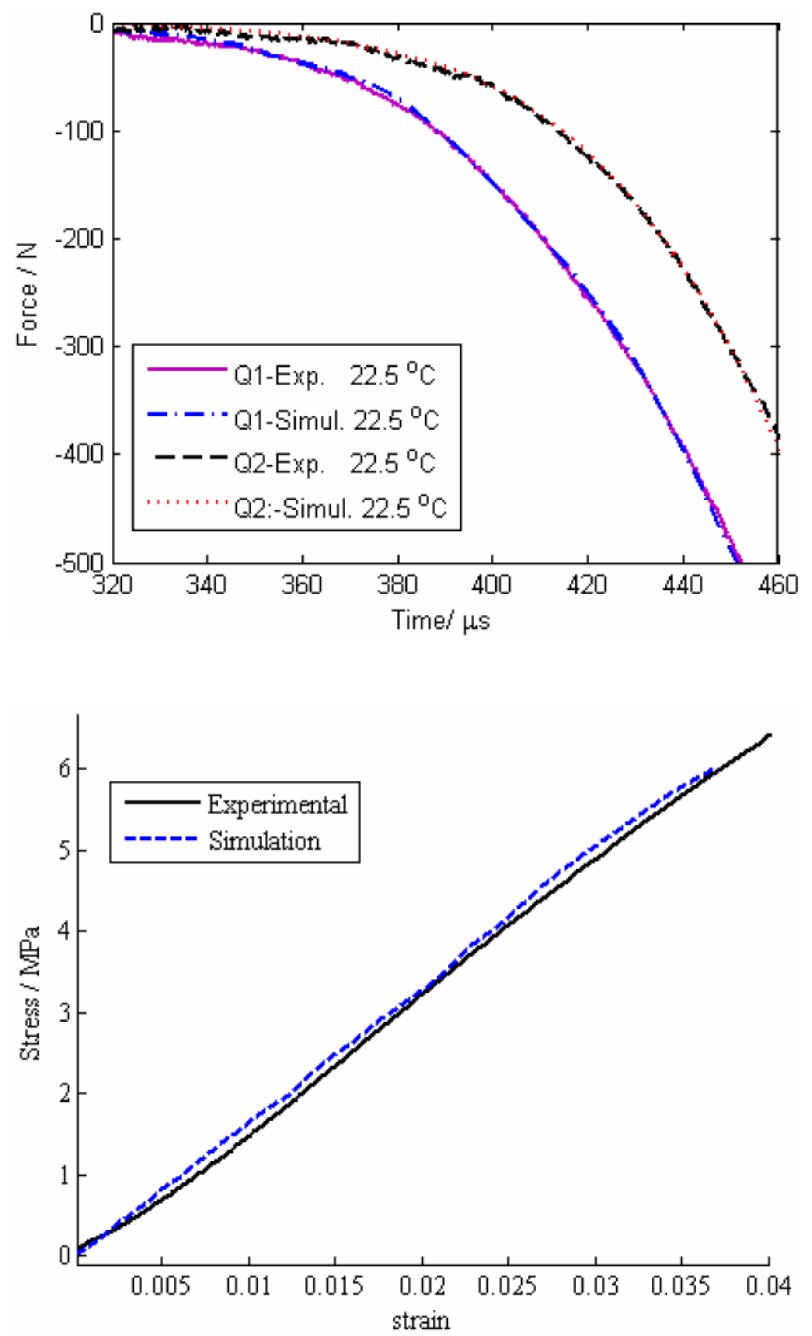

measurements. A further confirmation of this recognition is the comparison of the computed and measured force in Fig. 16 which is associated with the strain rate history in Fig. 14.

We reiterate that the test for the applicability of the time-temperature shifted, quasistatically obtained master curve is the degree to which this quasistatic data reproduces computationally the measurements in dynamic situations. Because there were limitations to obtaining quasistatic measurements at and above room temperature as well as below the glass transition (see Sect. 3), the time range accessed by the Hopkinson bar tests is correspondingly limited. We have, therefore performed comparison of dynamically recorded stress responses at five nominally different temperatures (room temperature, $0^{\circ} \mathrm{C},-20^{\circ} \mathrm{C},-35^{\circ} \mathrm{C}$ and $-39^{\circ} \mathrm{C}$ ) with the lowest two calling for some special discussion.

Regarding the tests at sub-room temperature conditions we start with the dynamic tests at $0^{\circ} \mathrm{C}$. Figure 17 shows again the comparison of the recorded data and the corresponding computed responses at the two quartz gages $(\mathrm{Q} 1$ is located on the incident bar and Q2 on the transmission bar). The agreement is very satisfactory, indicating that the data acquired 
Fig. 17 Comparison between experimental (quartz gage) and simulation results at $0^{\circ} \mathrm{C}$
Fig. 18 Comparison between measurements (quartz gages) and simulation results at $-20^{\circ} \mathrm{C}$
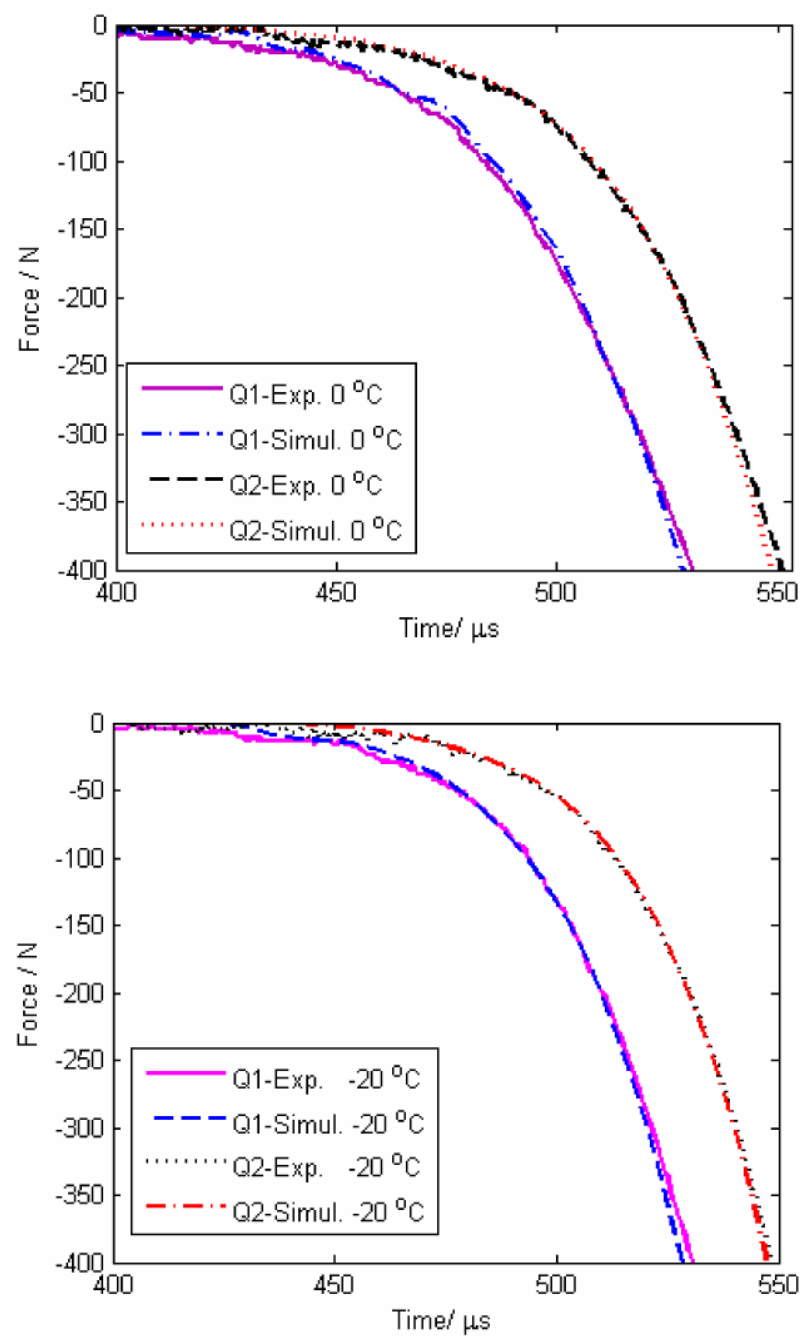

quasistatically in the time frame of 10 to $10^{4}$ seconds applies to the dynamic problem which proceeds in the time range on the order of $10^{-4}$ seconds (c.f. Fig. 8 recorded at room temperature).

Because there was relatively little change between the response at 0 and $-10^{\circ} \mathrm{C}$, we turn to the dynamic tests conducted at $-20^{\circ} \mathrm{C}$, illustrated in Fig. 18 . We note again that the comparison between computed and measured values at the two quartz gage locations are very favorable. A repeat of the same tests at nominally $-34^{\circ} \mathrm{C}$ yields the results in Fig. 19.

The comparison of the physical measurements with the computational model at (nominally) $-34^{\circ} \mathrm{C}$, and especially at the nominal $-39^{\circ} \mathrm{C}$ temperature requires some additional discussion. It will be recalled that the thermal control for the split Hopkinson bar (Fig. 6) provided thermal specification and determination only within a small range of temperatures, which range was estimated to be on the order of $1-3^{\circ} \mathrm{C}$. From Sect. 4.2 we note that the relaxation modulus becomes very sensitive to temperate changes at these low temperatures. As a result of these constraints it turns out that the computations for (nominally) $-34^{\circ} \mathrm{C}$ do 
Fig. 19 Comparison of measurements at nominally $-34.3^{\circ} \mathrm{C}$ with simulations at neighboring temperatures
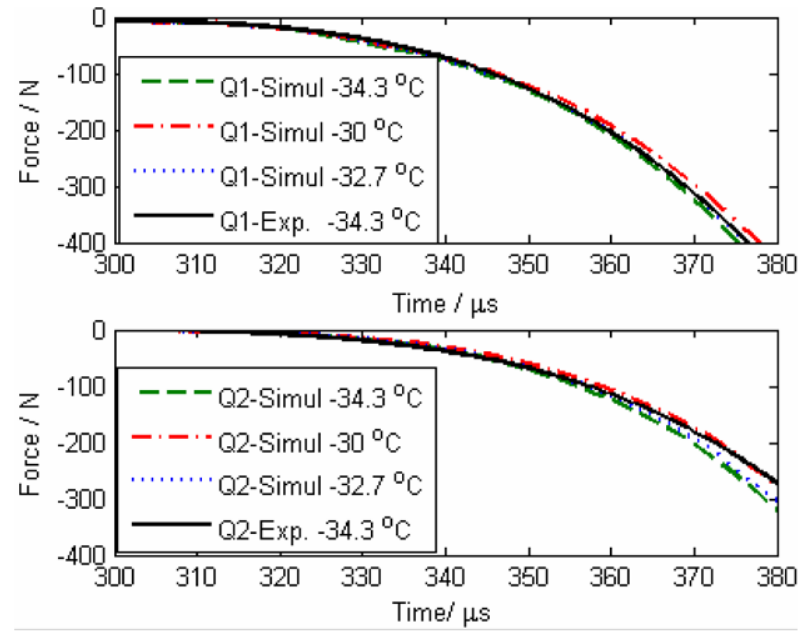

Fig. 20 Comparison of measurements at the nominal temperature of $-39^{\circ} \mathrm{C}$ and simulations at a neighboring temperature
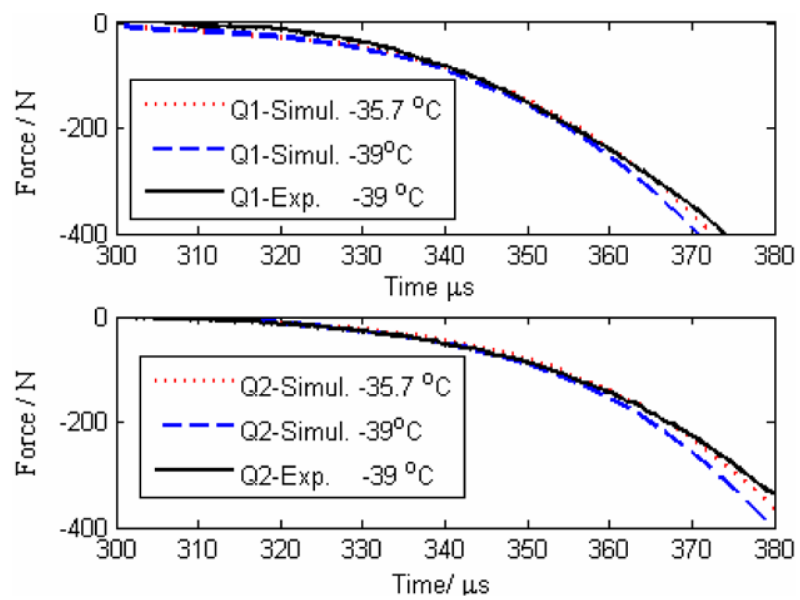

not provide as close a fit as the comparisons at the higher sub-zero temperatures if that value is assumed to represent the physical situation precisely. We find, however, that a computation for $-32.7^{\circ} \mathrm{C}$, which falls well within the range of the temperature uncertainty, provides a reasonably close fit. This is illustrated in Fig. 19, where results for the upper and lower values consistent with the experimental temperature range are also included.

The situation at $-39^{\circ} \mathrm{C}$ is more difficult yet, primarily because at this temperature even the small variations throughout the specimen that cannot be gauged, may influence the experimental result significantly. Moreover, at this temperature the dynamic response of the material involves time scales that are not directly represented in the relaxation modulus of Fig. 2; for this reason we judiciously extended the relaxation modulus at shorter times by a power law extension to shorter times. We note that the greatest consistency between experiment and computation corresponds to a temperature of $-35.7^{\circ} \mathrm{C}$, which is removed from the nominally measured temperature by more than the $3^{\circ} \mathrm{C}$ maximal deviation. It is clear that the experimental precision at this temperature range suffers, so that a clear demonstration of the results is compromised, though the computational replication of the measurements 
is still reasonable, if one accepts the error due to the lacking precision in prescribing the temperature of the specimen itself.

\section{Summary}

We have thus established that for dynamic strain rates achieved in a split Hopkinson (Kolsky) pressure bar and using (compound) pulse shaping, specimens will undergo equilibrium deformations during the loading process.

We find that the computational use of viscoelastic properties, determined quasistatically and with the aid of the time-temperature shifting process, renders results at various temperatures and at deformation rates that are consistent with wave propagation measurements in split Hokinson (Kolsky) bar experiments. Accordingly, we have established that for linearly viscoelastic behavior of a polyurea elastomer, obeying the time-temperature superposition principle, the (classical) viscoelastic master curve provides the proper material characterization for dynamic situations, in which strain rates exceed the time scales underlying the quasistatic characterization by factors of $10^{6}$ to $10^{8}$.

While the current evaluation applies strictly only to the material studied at hand, the extensive experience with material characterization and the arguments cited in the Introduction would encourage one to deduce, that this behavior is more one of principle rather than being very material specific. In closing this paper we reiterate, that when strains reach levels that generate nonlinearly viscoelastic behavior such an "assertion" is on a less firm foundation, but expectations may be justified that similar behavior holds at least approximately.

Acknowledgements We gratefully acknowledge the support of the Office of Naval Research for supporting this investigation under Grants N00014-05-1-0548 and N00014-05-1-0624. We thank Dr. R.S. Barsoum for his suggestions and discussions during the course of this research and value his recognition that this type of study had a longstanding need for resolution in the viscoelastic/polymer engineering community.

\section{References}

Chakkarapani, V., Ravi-Chandar, K., Liechti, K.M.: Characterization of multiaxial constitutive properties of rubbery polymers. J. Eng. Mater. Technol. 128, 489-494 (2006)

Chen, W., Lu, F., Frew, D.J., Forrestal, M.J.: Dynamic compression testing of soft materials. J. Appl. Mech. 69, 214-223 (2002)

Knauss, W.G., Zhao, J.: Improved relaxation time coverage in ramp-strain histories, Mech. Time-Depend Mater. (2008, this issue)

Treloar, L.R.G.: The Physics of Rubber Elasticity. Clarendon Press, Oxford (1975)

Tschoegl, N., Knauss, W.G., Emri, I.: The effect of temperature and pressure on the mechanical properties of thermo- and/or piezorheologically simple polymeric materials in thermodynamic equilibrium-A critical review. Mech. Time-Depend. Mater. 6, 53-99 (2002)

Williams, M.L., Landel, R.F., Ferry, J.D.: The temperature dependence of relaxation mechanisms in amorphous polymers and other glass-forming liquids. J. Am. Chem. Soc. 77, 3701-3706 (1955) 